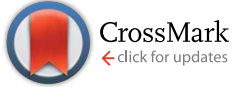

Cite this: RSC Adv., 2017, 7, 13749

Received 31st January 2017

Accepted 20th February 2017

DOI: $10.1039 / \mathrm{c} 7 \mathrm{ra01311f}$

rsc.li/rsc-advances

\section{Inside-fused perylenediimide dimers with planar structures for high-performance fullerene-free organic solar cells $\uparrow$}

\begin{abstract}
Guodong Ding, ${ }^{a}$ Ailing Tang, ${ }^{\text {*a }}$ Fan Chen, ${ }^{\mathrm{a}}$ Keisuke Tajima, ${ }^{\mathrm{b}}$ Bo Xiao ${ }^{\mathrm{a}}$ and Erjun Zhou ${ }^{\star a}$
For perylenediimide derivatives it seems that twisted structures are essential to avoid excessive aggregation tendencies and realize high-performance fullerene-free solar cells. However, in this communication, we designed and synthesized two planar inside-fused perylenediimide dimers, $\mathrm{TDI}_{2}$ and $\mathrm{BDT}^{-\mathrm{TDI}} \mathrm{I}_{2}$. Theoretical calculations reveal that both $\mathrm{TDI}_{2}$ and $\mathrm{BDT}^{-} \mathrm{TDI}_{2}$ have a highly planar molecular conformation with small dihedral angles of $0.02^{\circ}$ and $11.4^{\circ}$ between two TDI segments, respectively. By using BDDT as the donor polymer, power conversion efficiencies (PCEs) of the photovoltaic cells reached $5.80 \%$ for $\mathrm{TDI}_{2}$ and $4.52 \%$ for $\mathrm{BDT}-\mathrm{TDI}_{2}$, with high open-circuit voltages $\left(V_{\mathrm{OC}}\right)$ of $\sim 1.0 \mathrm{~V}$. These results indicate planar PDI-dimer derivatives are also possible electron acceptors to realize highperformance fullerene-free solar cells.
\end{abstract}

Polymer solar cells (PSCs) receive much attention. Perylenediimide (PDI)-based compounds have attracted extensive interest as alternatives to conventional fullerene derivatives in organic solar cells (OSCs) due to their high electron affinity, strong light absorption ability and high chemical stability. ${ }^{1-5}$ However, mono-PDI derivatives typically possess planar molecular structures, which result in large crystalline domains in donor-acceptor mixed films and poor photovoltaic performance in the early stage of the study. ${ }^{6-8}$ Thus, great efforts have been devoted to reduce the excessive aggregation tendency of PDI-based small molecules. Bulky alkyl chains and substituent groups have been introduced to modify the properties of PDI derivatives; however, limited improvement was achieved.,910 From 2013, twisted PDI-dimers, by linking two PDI units with various spacers (see Scheme 1A), have been developed, and has been proven as a promising strategy to reduce the intermolecular interactions and thus to hinder molecular aggregations. ${ }^{11-14}$ In addition, some non-planar, three-dimensional (3D) molecular structures containing multi-PDI segments, ${ }^{15-19}$ such as PDI-trimers and PDI-tetramers, were also designed and synthesized to effectively suppress self-aggregation and crystallization of the PDI units. The performance of OSCs based on these non-planar materials was consequently improved and the highest power conversion efficiency (PCE) of $8-9 \%$ has been

${ }^{a}$ CAS Key Laboratory of Nanosystem and Hierarchical Fabrication, CAS Center for Excellence in Nanoscience, National Center for Nanoscience and Technology, Beijing 100190, P. R. China.E-mail:zhouej@nanoctr.cn; tangal@nanoctr.cn

${ }^{b}$ Emergent Functional Polymers Research Team, RIKEN Center for Emergent Matter Science (CEMS), 2-1 Hirosawa, Wako 351-0198, Japan

$\dagger$ Electronic supplementary information (ESI) available. See DOI: 10.1039/c7ra01311f realized. However, this kind of linking method at the bay positions of PDI also leads to twisting of the perylene core, which disrupts the $\pi$-stacking of $\pi$-surface and thus diminishes the electron transport in the bulk state in the thin film.

To control the planar structure of PDI ring, an effective strategy is to fuse heteroatoms into the PDI carbon skeleton. ${ }^{20}$ Thus, from 2015, several groups developed various novel PDIbased electron acceptors by incorporating the heteroatoms, such as $\mathrm{S}$, Se and $\mathrm{N}$ atoms, at the outside bay positions of the PDI framework (see Scheme 1B). ${ }^{21-23}$ For these compounds, the fused PDI ring is highly planar, but the dihedral angels between two fused rings are as large as $\sim 80^{\circ}$. Promising photovoltaic performances were achieved with PCEs of $7.2 \%,{ }^{21} 8.4 \%$ (ref. 22) and $7.6 \%$ (ref. 23) for the S, Se and $\mathrm{N}$ annulated PDI derivatives respectively.

From the reports above, it seems that the twisted structures for PDI derivatives are essential to realize high-performance OSCs, including PDI-dimers, PDI-trimers, PDI-tetramers, outside-fused (by S, Se or N atoms) PDI-dimers or trimers, etc. However, this conclusion might be not totally correct because of the absence of planar PDI-dimers. Thus, it is highly desirable to

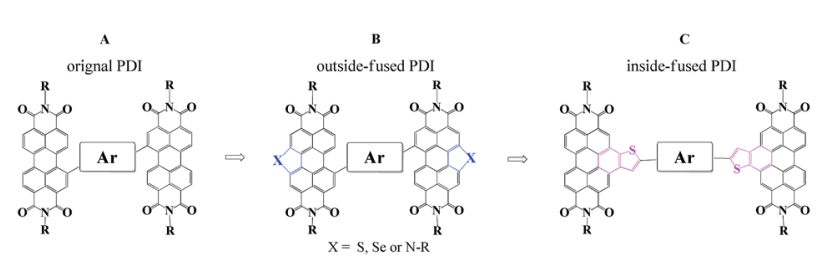

Scheme 1 Three classes of PDI based non-fullerene small molecule acceptors. 

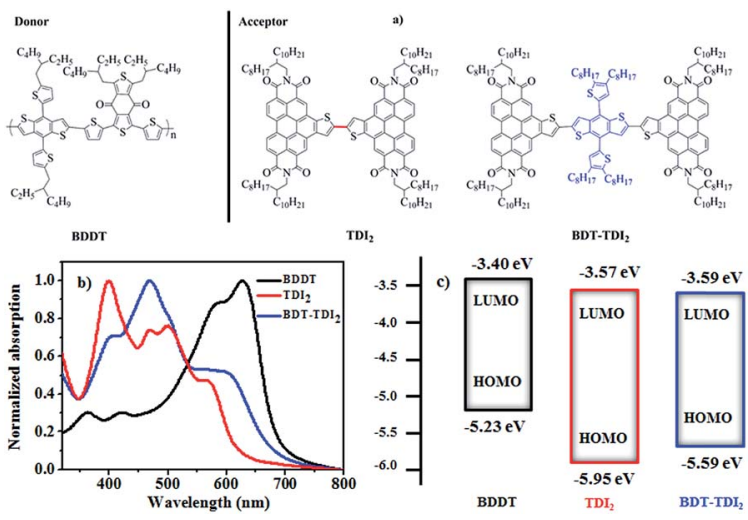

BDT-TDI
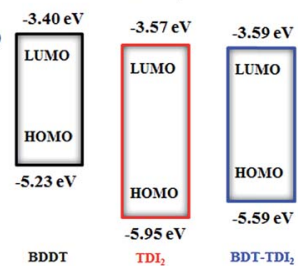

Fig. 1 (a) Donor and acceptor structure information used in this study; (b) normalized UV-vis absorption spectra of TDIs and BDDT in thin films; (c) Schematic energy level of TDIs and BDDT determined by cyclic voltammetry.

get this kind of materials, which will help to understand the relationships of structure-property-photovoltaic performance. However, it is a large challenge to realize planar PDI-dimer derivatives due to the large steric hindrance of $\mathrm{C}-\mathrm{H}$ band at the inside bay-positions. In this work, we designed and synthesized a kind of inside-fused PDI derivatives, thianaphthene fused perylenediimde (TDI)-based dimers (see Scheme 1C). The dimers of TDI have the potential to adopt a planar conformation from two aspects: (1) the large distance between two TDI segments and (2) small steric hindrance by removing the $\mathrm{H}$-atom at the bay-position of PDI segments. A similar strategy has been reported recently for naphthalene diimide (NDI)-based molecules, which could realize planar structures. ${ }^{24}$ Here, we synthesized two TDI-based molecules, one is directly linked two TDI building blocks, the other is linked two TDI by using classic benzo[1,2- $\left.b: 4,5-b^{\prime}\right]$ dithiophene (BDT) as spacer (see Fig. 1).

\section{Results and discussion}

The detailed synthetic methods of two TDI compounds and their characterizations by NMR and MALDI-TOF are shown in Schemes S1 and S2, Fig. S3 and S4 (ESI $\dagger$ ). The important intermediate, TDI-Br, was synthesized by the annulated of PDITh-Br, and $\mathrm{TDI}_{2}$ and $\mathrm{BDT}^{-} \mathrm{TDI}_{2}$ were synthesized via stille coupling reaction. This two molecules are highly soluble in common chlorinated solvents such as chloroform (CF), chlorobezene (CB) and $o$-dichlorobenzene (DCB) owning to the large alkyl chains in TDI building blocks, which is favorable for solution processed OSC devices.

In order to understand the electronic distribution and geometrical configuration of TDIs, theoretical calculations were performed by using the density functional theory (DFT) at the B3LYP/6-31G level. To simplify the calculations, the long alkyl chains were replaced with methyl groups. The simulated electron density distributions and the dihedral angles are illustrated in Fig. 2. As we expected, both the TDI-based molecules show more planar structures than original PDI-dimers or outside-fused PDI dimers. It is surprised that the dihedral angle between the two TDI groups in $\mathrm{TDI}_{2}$ is as small as $0.02^{\circ}$, although it slightly increased to $11.4^{\circ}$ for $\mathrm{BDT}^{-\mathrm{TDI}_{2}}$ molecule. The almost planar configuration between the two TDI groups in $\mathrm{TDI}_{2}$ ascribe to the strong conjugation effect between $\pi$ electrons in TDI rings which makes the single $\mathrm{C}-\mathrm{C}$ bond into almost planar $\mathrm{C}=\mathrm{C}$ bond (Fig. 2a). This will lead to more effective conjugation, which is favourable to get higher carrier mobility.

To investigate the photovoltaic performance, the proper selection of energy matched donor materials is also very critical for making high-PCE solar cells. Due to well energy level matches and relatively complementary absorption spectra, BDDT was chose as donor polymer ${ }^{25}$ in the present study. The UV-vis absorption spectra of TDIs and the polymer BDDT in thin films on quartz substrates are presented in Fig. $1 \mathrm{~b}$, and the spectra in $\mathrm{CH}_{2} \mathrm{Cl}_{2}$ solution $\left(\sim 10^{-5} \mathrm{~mol} \mathrm{~L}^{-1}\right)$ are shown in Fig. S1 $(\mathrm{ESI}+)$. Because of the highly planar conformation of $\mathrm{TDI}_{2}$, it shows high strong absorption in the region of 320-640 $\mathrm{nm}$ in $\mathrm{CH}_{2} \mathrm{Cl}_{2}$ solution, with a maximum molar extinction coefficient of $6.9 \times 10^{4} \mathrm{~L} \mathrm{~mol}^{-1} \mathrm{~cm}^{-1}$ at $391 \mathrm{~nm}$. BDT-TDI 2 has an obvious red-shift with a maximum molar extinction coefficient of $8.3 \times$ $10^{4} \mathrm{~L} \mathrm{~mol}^{-1} \mathrm{~cm}^{-1}$ at $469 \mathrm{~nm}$. From Fig. S1† and $1 \mathrm{~b}$, it also indicates the new TDI compounds aggregated in dilute solution, mainly due to the unique high planar configuration. Obviously, the polymer donor and the TDIs acceptors can form a relatively complementary absorption spectra in the region of
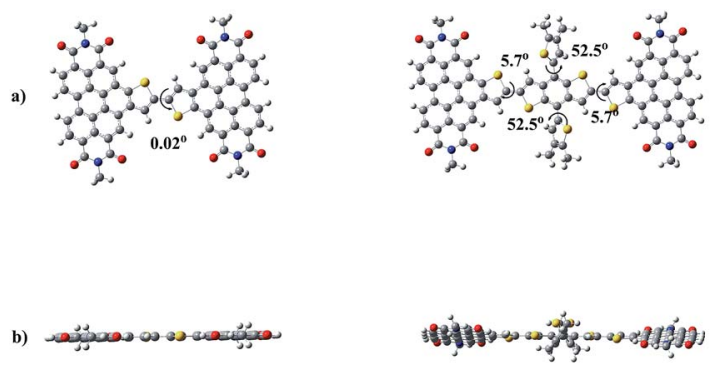

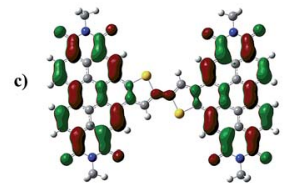

LUMO: $-3.62 \mathrm{eV}$

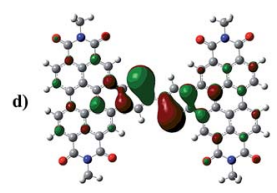

HOMO: $-6.11 \mathrm{eV}$

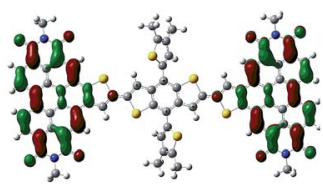

LUMO: $-3.49 \mathrm{eV}$

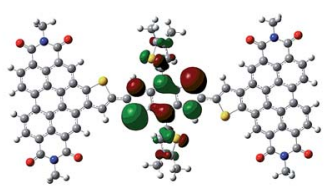

HOMO: $-\mathbf{5 . 5 4} \mathrm{eV}$
Fig. 2 (a) Top view and (b) side view of the optimized geometries for TDIs; calculated electron density distributions of (c) LUMO and (d) HOMO for TDIs by DFT at the B3LYP/6-31G level. 

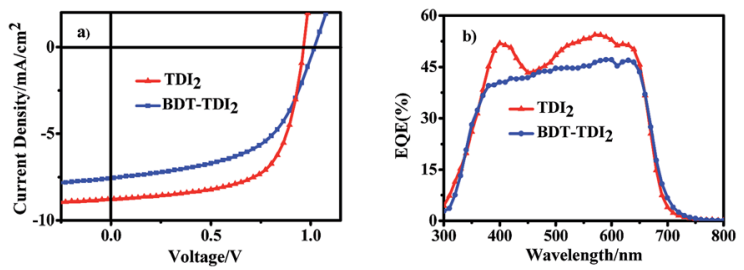

Fig. 3 (a) J-V curves of solar cells based on BDDT and TDIs blend films under optimized conditions and (b) the corresponding EQE spectra.

320-720 nm, which makes the material combination can utilize more sunlight.

Electrochemical cyclic voltammetry (CV) was used to investigate the electronic structures and the energy levels (Fig. 1c). The highest occupied molecular orbital (HOMO) and the lowest unoccupied molecular orbital (LUMO) levels calculated from the onset of oxidation and reduction potentials are summarized in Table S1. $\dagger$ The LUMO levels of $\mathrm{TDI}_{2}$ and BDT-TDI ${ }_{2}$ are -3.57 and -3.59 respectively, higher-lying than those of PCBM $(\sim-3.9$ eV), PDI-dimers $(\sim-4.04 \mathrm{eV})^{14}$ and outside-fused PDI-dimers $(\sim-3.85 \mathrm{eV}),{ }^{21-23}$ which should contribute to a higher $V_{\mathrm{OC}}$.

To evaluate the photovoltaic performance of the new TDIs materials, conventional $\mathrm{BHJ}$ OSCs were fabricated with the ITO/PEDOT:PSS/BDDT:TDIs/Ca/Al structure. The fabrication process of the device is described in detail in ESI. $\dagger$ The current density-voltage $(J-V)$ curves and corresponding device parameters measured under AM $1.5 \mathrm{G}$ illumination $\left(100 \mathrm{~mW} \mathrm{~cm}^{-2}\right)$ are summarized in Fig. 3a and Tables S2, S3, $\dagger$ and the optimized data are presented in Table 1. As expected, TDIs-based device exhibits a $V_{\mathrm{OC}}$ much higher than $1.0 \mathrm{~V}$. From the Tables $\mathrm{S} 2$ and $\mathrm{S} 3, \uparrow$ it can be found obviously that chlorobenzene (CB) and $o$ dichlorobenzene (ODCB) is much better choice than chloroform (CF). It is also known that the weight ratios of donor and acceptor in active layers play important roles in the performance of OSCs. Thus, five different weight ratios of $1: 2,1: 1.5$, 1.1, $1.5: 1$ and $2: 1(\mathrm{w} / \mathrm{w})$ for BDDT : TDIs blend films were examined. To further optimize the device performance, a small amount of additive (1,8-diiodooctane (DIO), 1-chloronaphthalene (CN) or diphenyl ether (DPE)) was used to optimize the active layer morphology. The results showed that both the weight ratio and additive concentration play important roles in determining device performance. Finally, the best power

Table 1 Photovoltaic performance of the optimized devices based on BDDT : TDIs ( $1: 1, \mathrm{w} / \mathrm{w})$ under the illumination of AM $1.5 \mathrm{G}, 100 \mathrm{~mW}$ $\mathrm{cm}^{-2}$

\begin{tabular}{llllll}
\hline Active layer & Solvent & $V_{\mathrm{OC}}(\mathrm{V})$ & $\begin{array}{l}J_{\mathrm{SC}} \\
\left.(\mathrm{mA} \mathrm{cm})^{-2}\right)\end{array}$ & $\mathrm{FF}$ & $\begin{array}{l}\text { PCE } \\
\max \left(\mathrm{ave}^{a}\right)\end{array}$ \\
\hline $\mathrm{TDI}_{2}$ & $\begin{array}{l}\mathrm{CB} / \mathrm{DPE} \\
(97: 3, \mathrm{v} / \mathrm{v})\end{array}$ & 0.96 & 8.78 & 0.65 & $\begin{array}{l}5.80 \% \\
(5.74 \%)\end{array}$ \\
BDT-TDI $_{2}$ & $\begin{array}{l}\mathrm{CB} / \mathrm{CN} \\
(99.8: 0.2, \mathrm{v} / \mathrm{v})\end{array}$ & 1.02 & 7.55 & 0.54 & $\begin{array}{l}4.52 \% \\
(4.40 \%)\end{array}$
\end{tabular}

${ }^{a}$ The average data measured by 5 devices. conversion efficiency up to $5.80 \%$ was achieved for BDDT:TDI 2 device, while for BDDT:BDT-TDI ${ }_{2}$ devices, optimized efficiency $4.52 \%$ was obtained.

External quantum efficiency (EQE) plots of the devices based on BDDT:TDIs processed with optimized conditions are shown in Fig. 3b. The devices show broad photocurrent response in 300-700 nm, which should be attributed to the absorptions of both BDDT and TDIs. The maximum EQE value of BDDT:TDI ${ }_{2}$ reached $54.4 \%$ at $570 \mathrm{~nm}$. The maximum EQE value for BDDT:BDT-TDI ${ }_{2}$ was $47.2 \%$ at $600 \mathrm{~nm}$, which is in accordance with the lower power conversion efficiency.

Two-dimensional grazing incidence wide angle X-ray scattering (2D GIWAXS) was measured to understand the crystallinity and orientation of both the polymer donor BDDT and the acceptors TDIs in the pristine and blend film, as shown in Fig. 4. As previously reported, BDDT was oriented in a face-on manner in the pristine polymer film. ${ }^{25}$ In contrast, TDIs film shows no obvious edge-on or face-on orientations probably because of its long alkyl chain which could disturb the molecular crystallinity. This result coincides with the XRD patterns of pristine TDIs film, where no obvious peaks could be found (Fig. S2†). From Fig. 4, BDDT chains in the blend films mainly oriented in a face-on manner, judging from the peaks of $\pi-\pi$ stacking remaining in the out-of-plane direction. This suggests that, the crystallinity and the orientation of BDDT shown in the pristine films were maintained. The blend films with preferential face-on orientation is favourable for OSC application which facilitates the hole transport. Moreover, the crystalline nature of BDDT even in the blend film should favourably contribute to the charge separation at the interface between TDIs and thus the high photoresponse in the long-wavelength region.

For BHJ solar cells, the thin-film morphology of the active layer determines the efficiency of charge separation and collection. To further gain insight into the surface morphology of the BHJ active layer, atomic force microscopy (AFM) in the tapping mode was carried out. As shown in Fig. 5, for all optimized BDDT:TDIs blend films, there are obvious phase
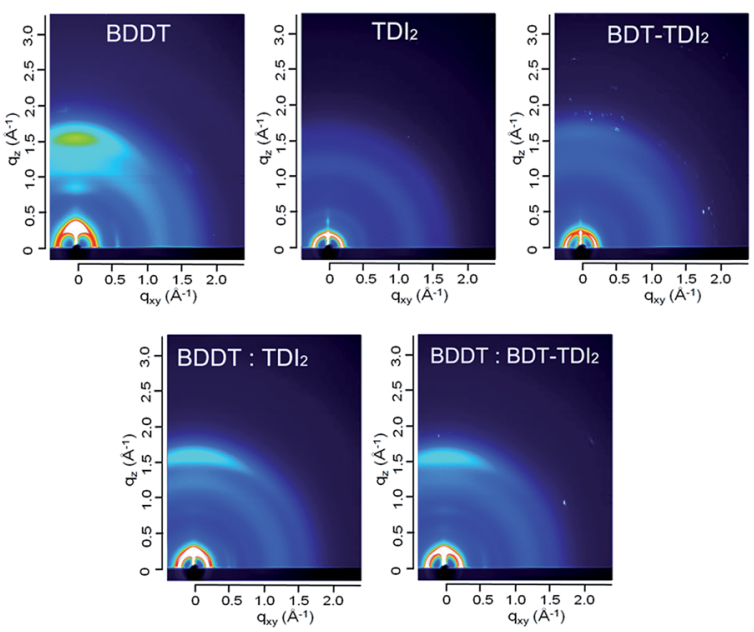

Fig. 4 2D GIWAXS patterns of the pristine films and the blend films. 
BDDT:BDT-TDI ${ }_{2}$
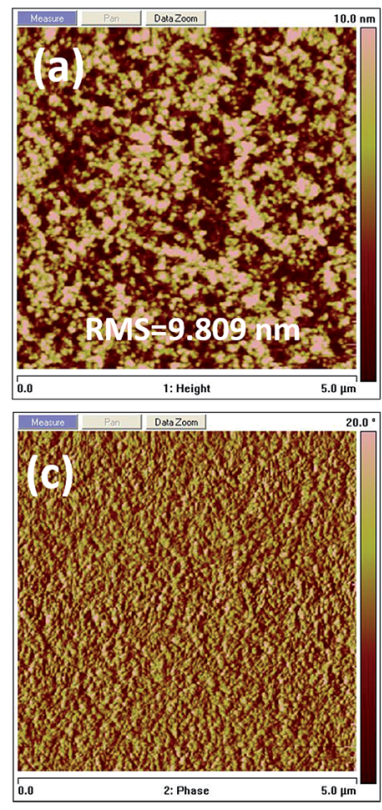

BDDT:TDI,
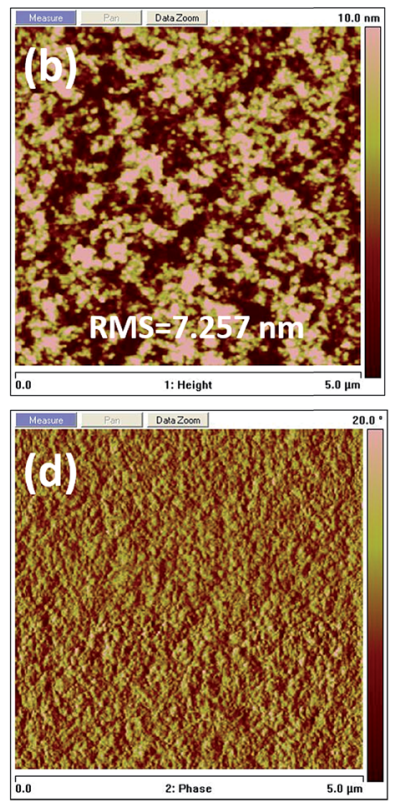

Fig. 5 AFM height (top) and phase (bottom) images $(5 \mu \mathrm{m} \times 5 \mu \mathrm{m})$ of BDDT:TDIs active layers. (a) and (c) BDDT:BDT-TDI 2 blend film, $3 \%$ DPE as additive (b) and (d) BDDT:TDI ${ }_{2}$ blend film, $0.2 \% \mathrm{CN}$ as additive.

separation happened and root mean square (RMS) roughness are $9.8 \mathrm{~nm}$ and $7.3 \mathrm{~nm}$ respectively. This appropriate phase separation and fibrous morphology of blend films may be beneficial to form the bicontinuous and interpenetrating networks required for efficient charge transport, resulting in the high PCEs.

In summary, we have designed and synthesized inside-fused PDI derivatives as small molecular electron acceptor materials for fullerene-free solar cells. The backbone of TDI is highly planar conformation compared to the original or outside-fused PDI framework. The new TDI-based molecules showed high strong absorption in the $320-650 \mathrm{~nm}$ region. The relationship between molecular structure, thin-film morphology, and device performance was systematically investigated by a combination of measurements. The highest power conversion efficiency (PCE) up to $5.80 \%$ was obtained with higher $V_{\mathrm{OC}}$ of $\sim 1.0 \mathrm{~V}$ when BDDT:TDI ${ }_{2}$ blend film device was fabricated. These results indicate that, besides twisted-structured PDI derivatives, planar PDI-dimer derivatives also have great potential to obtain satisfactory performances. Through delicate structure design, they are also possible electron acceptor to realize high-performance fullerene-free solar cells.

\section{Acknowledgements}

The authors thank the support from the National Natural Science Foundation of China (No. 51673048, 51473040, 21602040), the National Natural Science Foundation of Beijing (No. 2162045), and the Chinese Academy of Sciences (QYZDBSSW-SLH033). 2D GIWAXS experiments were performed at beam line BL46XU of SPring-8 with the approval of the Japan
Synchrotron Radiation Research Institute (JASRI) (Proposal 2016B1875) with the support by Dr Tomoyuki Koganezawa.

\section{Notes and references}

1 C. W. Tang, Appl. Phys. Lett., 1986, 48, 183.

2 X. Zhan, A. Facchetti, S. Barlow, T. J. Marks, M. A. Ratner, M. R. Wasielewski and S. R. Marder, Adv. Mater., 2011, 23, 268-284.

3 X. Zhao and X. Zhan, Chem. Soc. Rev., 2011, 40, 37283743.

4 Y. Lin, Y. Li and X. Zhan, Chem. Soc. Rev., 2012, 41, 42454272 .

5 Y. Lin and X. Zhan, Mater. Horiz., 2014, 1, 470-488.

6 L. Schmidt-Mende, A. Fechtenkötter, K. Müllen, E. Moons, R. H. Friend and J. MacKenzie, Science, 2001, 293, 11191122.

7 L. Sicot, B. Geffroy, A. Lorin, P. Raimond, C. Sentein and J.-M. Nunzi, J. Appl. Phys., 2001, 90, 1047-1054.

8 V. Kamm, G. Battagliarin, I. A. Howard, W. Pisula, A. Mavrinskiy, C. Li, K. Müllen and F. Laquai, Adv. Energy Mater., 2011, 1, 297-302.

9 P. E. Hartnett, A. Timalsina, H. S. Matte, N. Zhou, X. Guo, W. Zhao, A. Facchetti, R. P. Chang, M. C. Hersam, M. R. Wasielewski and T. J. Marks, J. Am. Chem. Soc., 2014, 136, 16345-16356.

10 Y. Cai, L. Huo, X. Sun, D. Wei, M. Tang and Y. Sun, Adv. Energy Mater., 2015, 5, 1500032.

11 J. Zhao, Y. Li, H. Lin, Y. Liu, K. Jiang, C. Mu, T. Ma, J. Y. L. Lai, H. Hu, D. Yu and H. Yan, Energy Environ. Sci., 2015, 8, 520-525.

12 Q. Yan, Y. Zhou, Y.-Q. Zheng, J. Pei and D. Zhao, Chem. Sci., 2013, 4, 4389.

13 X. Zhang, Z. Lu, L. Ye, C. Zhan, J. Hou, S. Zhang, B. Jiang, Y. Zhao, J. Huang, S. Zhang, Y. Liu, Q. Shi, Y. Liu and J. Yao, Adv. Mater., 2013, 25, 5791-5797.

14 Y. Zang, C. Z. Li, C. C. Chueh, S. T. Williams, W. Jiang, Z. H. Wang, J. S. Yu and A. K. Jen, Adv. Mater., 2014, 26, 5708-5714.

15 Y. Lin, Y. Wang, J. Wang, J. Hou, Y. Li, D. Zhu and X. Zhan, Adv. Mater., 2014, 26, 5137-5142.

16 Y. Liu, C. Mu, K. Jiang, J. Zhao, Y. Li, L. Zhang, Z. Li, J. Y. Lai, H. Hu, T. Ma, R. Hu, D. Yu, X. Huang, B. Z. Tang and H. Yan, Adv. Mater., 2015, 27, 1015-1020.

17 J. Lee, R. Singh, D. H. Sin, H. G. Kim, K. C. Song and K. Cho, Adv. Mater., 2016, 28, 69-76.

18 Q. Wu, D. Zhao, A. M. Schneider, W. Chen and L. Yu, J. Am. Chem. Soc., 2016, 138, 7248-7251.

19 Y. Duan, X. Xu, H. Yan, W. Wu, Z. Li and Q. Peng, Adv. Mater., 2017, 29, 1605115.

20 H. Qian, W. Yue, Y. Zhen, S. Di Motta, E. Di Donato, F. Negri, J. Qu, W. Xu, D. Zhu and Z. Wang, J. Org. Chem., 2009, 74, 6275-6282.

21 D. Sun, D. Meng, Y. Cai, B. Fan, Y. Li, W. Jiang, L. Huo, Y. Sun and Z. Wang, J. Am. Chem. Soc., 2015, 137, 1115611162. 
22 D. Meng, D. Sun, C. Zhong, T. Liu, B. Fan, L. Huo, Y. Li, W. Jiang, H. Choi, T. Kim, J. Y. Kim, Y. Sun, Z. Wang and A. J. Heeger, J. Am. Chem. Soc., 2016, 138, 375-380.

23 A. D. Hendsbee, J.-P. Sun, W. K. Law, H. Yan, I. G. Hill, D. M. Spasyuk and G. C. Welch, Chem. Mater., 2016, 28, 7098-7109.
24 W. Chen, M. Nakano, J.-H. Kim, K. Takimiya and Q. Zhang, J. Mater. Chem. C, 2016, 4, 8879-8883.

25 L. Ye, X. Jiao, W. Zhao, S. Zhang, H. Yao, S. Li, H. Ade and J. Hou, Chem. Mater., 2016, 28, 6178-6185. 